\title{
REDUCED-COMPLEXITY MULTI-STAGE BLIND CLUSTERING EQUALISER
}

\author{
S. Chen, S. McLaughlin, P.M. Grant and B. Mulgrew \\ Department of Electrical Engineering, University of Edinburgh \\ King's Buildings, Edinburgh EH9 3JL, UK
}

\begin{abstract}
A multi-stage blind clustering algorithm is proposed for equalisation of $M$-QAM channels. A novel hieranchical decomposition divides the overall task of equalising a highorder QAM channel into much simpler sub-tasks. Each subtask can be accomplished fast and reliably using a blind clustering algorithm derived originally for 4-QAM signals. The well-known constant modulus algorithm (CMA) is used as a benchmark to assess this novel multi-stage blind equaliser and it is demonstrated that the new blind adaptive algorithm achieves much faster convergence. This multi-stage clustering equaliser only requires slightly more computations than the very simple CMA and, like the latter, its computational complexity does not increase as the levels of digital symbols increase.
\end{abstract}

\section{Introduction}

Blind equalisation techniques for nonminimum phase channels can loosely be classified into three categories. The first class of blind equalisation algorithms constructs a transversal equaliser directly to unravel the effects of channel impulse response. This class includes many well-known blind equalisers [1]-[7], which are often referred to as Bussgang algorithms. Most of practical emphasis has been focused on the schemes known as Godard's algorithms. In particular, the CMA has a very simple computational requirement and is widely applied to high-order QAM signals [7],[8]. The second class of blind equalisation algorithms identifies a channel model using techniques based on higher order cumulants [9]-[11]. Once a channel impulse response is obtained, it can then be employed to design an equaliser. This second class of blind equalisers, although very general and powerful, requires extensive computation. Recently, blind equalisation algorithms based on the maximum likelihood sequence estimation or the Bayesian sequence estimation have been proposed [12]-[14]. This third class of blind equalisers typically employs some joint channel and data estimation technique, which is computationally very expensive. An advantage of this third approach is that relatively few signal samples are required to achieve equalisation objective.

In the ICC'92, Karaoguz and Ardalan [15] presented a blind equaliser based on maximizing the a-posteriori (MAP) probability density function (p.d.f.) of the transversal equaliser output subject to the equaliser weights. They applied this new blind algorithm to 4-QAM signals and demonstrated its superior performance over some well-known blind equalisers. It should be emphasized that the meaning of MAP here is quite different from that of the MAP detector for known channels [16] and its blind approximation [13]. Karaoguz and Ardalan's algorithm, referred to as the soft decision-directed blind algorithm in [15], is a Bussgang-type algorithm well fitted to the finite nature of digital symbol constellation. To avoid any confusion, this algorithm will be referred to as the blind clustering algorithm.

The present study extends Karaoguz and Ardalan's blind clustering algorithm to $M-\mathrm{QAM}(M>4)$ signals. A straightforward application of this blind algorithm to $M-\mathrm{QAM}$ signals may not be a good idea and this is explained in simple geometric terms. Such a direct extension can further encounter the difficulty of increased complexity. By decomposing the task of reconstructing the data constellation into appropriate sub-tasks, a multi-stage blind clustering procedure is derived. Because each sub-task is very easy to achieve, this blind adaptive algorithm can accomplish fast convergence. Furthermore, its complexity does not increase as the levels of the data increase, and is always equal to that of the 4-QAM case. The performance of the multi-stage blind clustering equaliser is compared with that of the popular CMA using simulated channels. The results obtained confirm the significantly improved performance of this new blind equaliser over the CMA.

\section{Blind equalisation}

Consider the baseband model of a digital communication channel characterized by a finite impulse response (FIR) filter and an additive white noise source. The received signal is given by

$r(k)=\sum_{i=0}^{n-1} a_{i} s(k-i)+e(k)$

where $n$ is the length of the channel impulse response, $a_{i}=a_{i R}+j a_{i l}$ are the complex channel tap weights, the complex symbol sequence $s(k)=s_{R}(k)+j s_{l}(k)$ is assumed to be independently identically distributed (i.i.d.), $e(k)=$ $e_{R}(k)+j e_{l}(k)$ is an i.i.d. complex Gaussian white noise with $\mathrm{E}\left[e_{R}^{2}(k)\right]=\mathrm{E}\left[e_{I}^{2}(k)\right]=\sigma_{e}^{2}$, and $\mathrm{E}[\cdot]$ denotes the expectation operator. The symbol constellation is $M-\mathrm{QAM}$ defined by

$s_{i l}=(2 i-Q-1)+j(2 l-Q-1), 1 \leq i, l \leq Q$.

where $Q=\sqrt{M}=2^{L}$, and $L$ is an integer.

To remove the channel distortion, an equaliser is employed. No training sequence is available to the equaliser and, furthermore, the channel is nonminimum phase. The equaliser

This work was supported by the UK Science and Engineering Research Council under award

GR/G/53095. SM gratefully acknowledges financial support from the UK Royal Society. 
has a FIR structure defined as

$y(k)=\sum_{i=0}^{m-1} w_{i} r(k-i)$

where $m$ is the order of the equaliser and is assumed to be large enough, and $w_{i}=w_{i R}+j w_{i l}$ are the complex equaliser weights. The equaliser has a delay $d \sim m / 2$.

A family of blind adaptive algorithms called Godard's algorithms [2] adjusts the equaliser weights by minimizing the nonconvex cost function

$\bar{J}_{q}(\mathbf{w})=\mathrm{E}\left[\left(|y(k)|^{q}-\Delta_{q}\right)^{2}\right]$,

using a stochastic gradient algorithm, where $\mathbf{w}=\left[w_{0}\right.$ $\left.\cdots w_{m-1}\right]^{T}$ is the equaliser weight vector, $q$ is a positive integer, and $\Delta_{q}$ is a positive real constant defined by $\Delta_{q}=\mathrm{E}\left[|s(k)|^{2 q}\right] / \mathrm{E}\left[|s(k)|^{q}\right]$. The case of $q=2$ is known as the CMA [3], which is by far the most popular blind equaliser for high-order QAM signal constellations. It has a very simple computational complexity, comparable to that of the least mean square algorithm.

\section{The blind clustering algorithm}

Assume for the time being that the equaliser weights have been correctly chosen. Then the equaliser output can be expressed in two terms

$y(k)=x(k)+v(k)$,

where $x(k)=s(k-d)$, and $v(k)$ is approximately a Gaussian white noise. Thus when the equalisation is accomplished, the equaliser output can be modelled approximately by $M$ Gaussian clusters with means

$y_{i l}=s_{i l}, 1 \leq i, l \leq Q$.

and an approximate covariance

$$
\left[\begin{array}{cc}
\mathrm{E}\left[v_{R}^{2}(k)\right] & \mathrm{E}\left[v_{R}(k) v_{I}(k)\right] \\
\mathrm{E}\left[v_{I}(k) v_{R}(k)\right] & \mathrm{E}\left[v_{I}^{2}(k)\right]
\end{array}\right] \approx\left[\begin{array}{cc}
\rho & 0 \\
0 & \rho
\end{array}\right]
$$

Denote the a priori probability of $y_{q l}$ as $p_{q l}$. Under the conditions given previously, the a-posteriori p.d.f. of $y(k)$ is

$\eta(\mathbf{w}, y(k))=\sum_{q=1}^{Q} \sum_{l=1}^{Q} \frac{p_{q l}}{2 \pi \rho} \exp \left(-\frac{\left|y(k)-y_{q l}\right|^{2}}{2 \rho}\right)$.

When the equaliser weights have not yet been set correctly, $y(k)$ does not satisfy the model (8) of $M$ Gaussian clusters. However, from (8), a bootstrap optimization process can be formed to adapt the equaliser. The equaliser weights are adjusted by maximizing the criterion

$\bar{\eta}(\mathbf{w})=\mathrm{E}[\boldsymbol{\eta}(\mathbf{w}, y(k))]$.

The resulting equaliser can be referred to as the blind clustering equaliser. Because all the $p_{q l}, 1 \leq q, l \leq Q$, can be assumed to be equal, the criterion (9) is equivalent to

$\bar{J}_{c}(\mathbf{w})=\mathrm{E}\left[J_{c}(\mathbf{w}, y(k))\right]$

with

$J_{c}(\mathbf{w}, y(k))=\rho \sum_{q=1}^{Q} \sum_{l=1}^{Q} \exp \left(-\frac{\left|y(k)-y_{q l}\right|^{2}}{2 \rho}\right)$.
In practice, the equaliser weights are adapted according to the following stochastic gradient algorithm

$w_{i}(k+1)=w_{i}(k)+\mu \frac{\partial J_{c}(\mathbf{w}(k), y(k))}{\partial w_{i}}$,

where $0 \leq i \leq m-1$. Karaoguz and Ardalan [15] applied this blind equaliser to 4-QAM channels.

A direct application of this algorithm to high-order QAM signals has some serious drawbacks. This is not just because the complexity of the algorithm increases as $M$ increases. Consider the simplest case depicted in Fig.1, the case of a real channel and 2-ary PAM symbols. The gradient of $J_{c}$ in this case becomes

$$
\begin{aligned}
\frac{\partial J_{c}}{\partial w_{i}}= & \left(-\exp \left(-\frac{\left(y(k)-s_{1}\right)^{2}}{2 \rho}\right)\left(y(k)-s_{1}\right)\right. \\
& \left.-\exp \left(-\frac{\left(y(k)-s_{2}\right)^{2}}{2 \rho}\right)\left(y(k)-s_{2}\right)\right) r(k-i) .
\end{aligned}
$$

Referring to Fig.1, when the equaliser output $y$ is closer to $s_{2}$, the algorithm adjusts the weights in favour of $s_{2}$ as reflected in the second term of (13). But it is possible that the correct decision may actually be $s_{1}$, and the algorithm also adjusts the weights towards this decision as indicated by the first term in (13). It is clear that the algorithm is trying to find a compromise for the two conflicting factors.

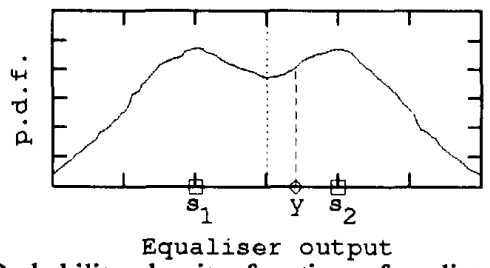

Fig.1. Probability density function of a distorted 2-ary PAM signal before equalisation is achieved.

In this simplest case and in the 4-QAM case, it is relatively easy for the algorithm to achieve a correct equalisation. For higher order QAM signals, there are more conflicting factors to consider and convergence speed will generally be slow. Other Bussgang algorithms have similar problems, and this is why the CMA was applied to high-order QAM signals in the first place to alleviate some of the difficulties associated with high signal levels. This problem can also be viewed through the objective function (10). For a large $M, \vec{J}_{c}(w)$ can be highly nonconvex and, therefore, a gradient algorithm may have difficulties in finding a desired solution.

\section{The multi-stage blind clustering algorithm}

We propose a multi-stage blind clustering procedure for highorder QAM signals, which overcomes the difficulties associated with multiple signal levels. This blind equalisation procedure is best illustrated by considering the 16-QAM case, where the overall equalisation objective is decomposed into the following two stages.

In the first stage, a 4-cluster model is adopted with the 4 cluster centres being $\tilde{s}_{11}=-2-j 2, \tilde{s}_{12}=-2+j 2, \tilde{s}_{21}=2-j 2$ and $\tilde{s}_{22}=2+j 2$. The equaliser weights are adjusted using this equivalent "4-QAM" model through the gradient algorithm 
(12). This is analogous to the idea of the CMA which considers the signal constellation as though it has an equivalent constant modulus. However the objective here is to achieve a roughly correct classification of equaliser outputs into the 4 quadrants $I_{i}, 1 \leq i \leq 4$, in the complex plane while the CMA attempts to reach the eye-open solution at one go, the former being an easy task to accomplish while the latter being much harder to achieve. After this stage, if the equaliser output appears in the quadrant $I_{i}$, the correct symbol point is in $I_{i}$ with high probability.

At the second stage, the 16-cluster model is adopted with the 16 cluster means being the correct symbol points $s_{q l}$, $1 \leq q, l \leq 4$. This cluster model is divided into 4 sub-models, one for each quadrant $I_{i}$. The equaliser adaptation is carried out as follows. If the equaliser output $y(k)$ is in the quadrant $I_{i}$, the corresponding 4-cluster sub-model is used to adapt the equaliser weights via the gradient algorithm (12). The aim of this stage is to reach the eye-open solution. The rational thinking behind this strategy is self-evident. The equaliser adaptation is done correctly with high probability owing to the primary clustering of the previous stage. Therefore the correct equalisation solution can be achieved faster and more reliably.

This blind clustering procedure is upwardly extendable. In general, the task of $M-\mathrm{QAM}$ equalisation, where $M=2^{2 L}$, can be achieved using an $L$-stage blind clustering equaliser. Because the sub-task of each stage can be accomplished easily and reliably, the overall convergence of the equaliser is achieved faster and more reliably. A further advantage of this multi-stage blind clustering equaliser is that the complexity of the algorithm does not increase as the number of the symbol points $M$ increases, and is always comparable to the minimum complexity of the 4-QAM case. Since the equaliser weights are adapted using a 4-cluster sub-model at each recursion via the gradient algorithm (12), this multi-stage blind clustering algorithm is only slightly more complex in terms of computation compared with the very simple CMA.

The choice of $\rho$ for each adaptation stage should ensure a proper separation of the clusters. A good rule for selecting the value of $\rho$ is that $\rho$ should be less than half of the distance between two adjacent cluster centres. If the value of $\rho$ is too large, a desired separation among clusters may not be achieved. On the other hand, when a too small $\rho$ is used, the algorithm attemps to impose a very tight control in the size of clusters and may fail to do so. Apart from these two extreme cases, the performance of the algorithm does not critically depend on the value of $\rho$, and there exists a wide range of values for $\rho$ at each stage of the adaptation.

\section{Performance comparison}

The performance of the multi-stage blind clustering algorithm is compared with that of the CMA using simulation. Convergence rate and robustness of these two blind adaptive algorithms are examined. In all the simulation examples, the middle tap of the equaliser was initialized to the nonzero value $1+j 0$, and the rest of the equaliser weights were initialized to $0+j 0$.

The first example is a telephone channel taken from [17]. The channel impulse response is listed in Table 1, digital symbols are 16-QAM, and the noise power is $\sigma_{e}^{2}=-20 \mathrm{~dB}$. Received signals before equalisation are shown in Fig.2. The equaliser had 23 taps. The two-stage blind clustering algorithm used 1500 samples for the first stage with $\rho=1.8,2000$ samples for the second stage with $\rho=0.7$ and an adaptive gain $\mu=0.003$ for the both stages. The equaliser outputs after this blind adaptation of 3500 samples are depicted in Fig.3. As a comparison, the CMA was also employed to adapt the equaliser with an adaptive gain $\mu=0.00001$. After the adaptation of 10000 samples, the eye diagram was still closed. The equaliser outputs after the adaptation of 20000 samples are shown in Fig.4.

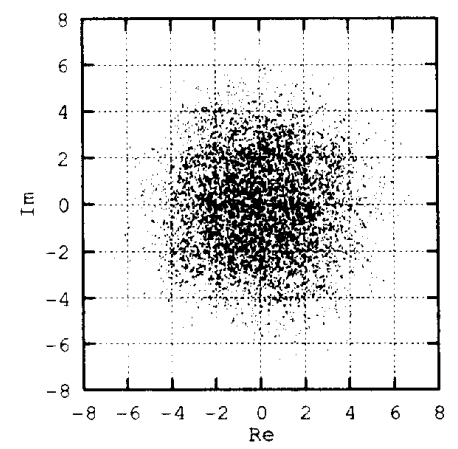

Fig.2. Observations of 16-QAM telephone channel.

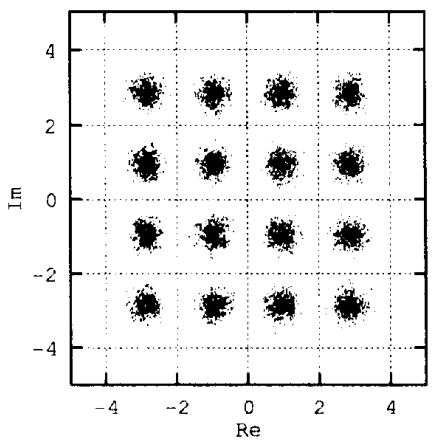

Fig.3. Outputs of two-stage blind clustering algorithm for 16-QAM telephone channel.

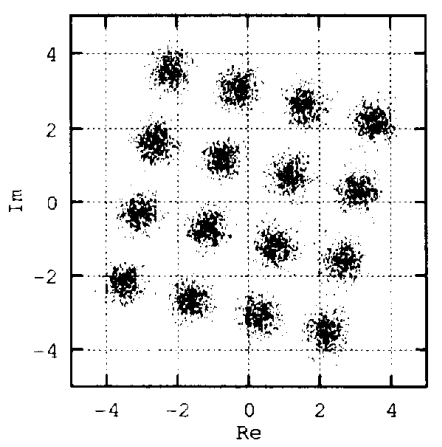

Fig.4. Outputs of CMA for 16-QAM telephone channel.

The second example is a 64-QAM microwave LOS fading channel operated at $24 \mathrm{Mbits} / \mathrm{s}$. The transmission pulse has a raised-cosine characteristics with a rolloff factor 0.5 and is split equally between the transmitter and receiver filters. A 


\begin{tabular}{|c|c|c|c|c|c|}
\hline Tap & Re & Im & Tap & Re & Im \\
\hline 0 & 0.0145 & -0.0006 & 11 & 0.0294 & -0.0049 \\
1 & 0.0750 & 0.0176 & 12 & -0.0181 & 0.0032 \\
2 & 0.3951 & 0.0033 & 13 & 0.0091 & 0.0003 \\
3 & 0.7491 & -0.1718 & 14 & -0.0038 & -0.0023 \\
4 & 0.1951 & 0.0972 & 15 & 0.0019 & 0.0027 \\
5 & -0.2856 & 0.1896 & 16 & -0.0018 & -0.0014 \\
6 & 0.0575 & -0.2096 & 17 & 0.0006 & 0.0003 \\
7 & 0.0655 & 0.1139 & 18 & 0.0005 & 0.0000 \\
8 & -0.0825 & -0.0424 & 19 & -0.0008 & -0.0001 \\
9 & 0.0623 & 0.0085 & 20 & 0.0000 & -0.0002 \\
10 & -0.0438 & 0.0034 & 21 & 0.0001 & 0.0006 \\
\hline
\end{tabular}

Table 1. A telephone channel impulse response.

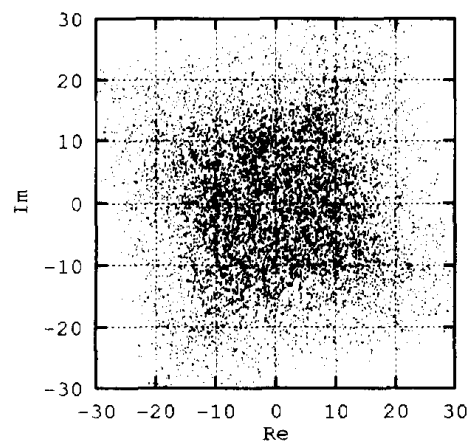

Fig.5. Observations of 64-QAM microwave channel.

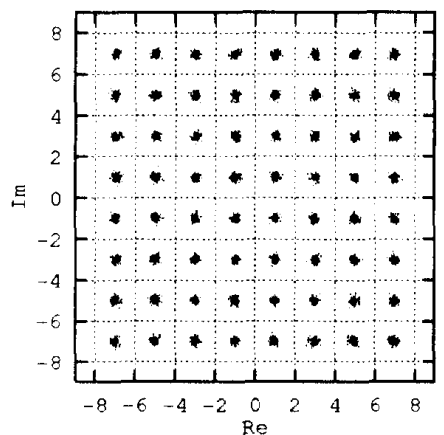

Fig.6. Outputs of three-stage blind clustering algorithm for 64-QAM microwave channel.

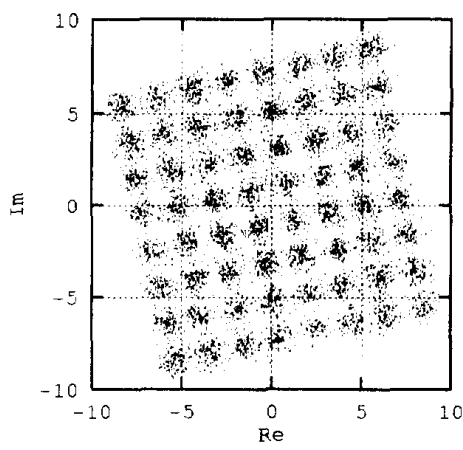

Fig.7. Outputs of CMA for 64-QAM microwave channel.

three-path Rayleigh fading channel is simulated with a fading rate $5 \mathrm{~Hz}$. The fading channel is implemented using the following tap-delay-line model

$v(t)=c_{0}(t) u(t)+c_{1}(t) u\left(t-\tau_{1}\right)+c_{2}(t) u\left(t-\tau_{2}\right)$. where $u(t)$ is the transmitter output and $v(t)$ is the fading channel output; $\tau_{1} \sim T_{s} / 10, \tau_{2} \sim T_{s} \beta$ and $T_{s}$ is the symbol duration; and the root mean powers of the both real and imaginary components of $c_{i}(t), 0 \leq i \leq 2$, are [0.7 0.60 .5$]$. The noise power is $\sigma_{e}^{2}=-20 \mathrm{~dB}$. Receiver outputs are sampled at symbol rate and passed to an equaliser of 9 taps. Received signals before equalisation are depicted in Fig.5. The three-stage blind clustering algorithm used 2000 samples for the stage one with $\rho=3.6,2000$ samples for the stage two with $\rho=1.8,1000$ samples for the stage three with $\rho=0.7$, and an adaptive gain $\mu=0.0003$ for all the three stages. After the above adaptation, the equaliser outputs are plotted in Fig.6 where, the stage-three adaptation was kept on. For the CMA. the adaptive gain had to be chosen as $\mu=0.1 \times 10^{-6}$ to avoid divergence. After an adaptation of 10000 samples, the equaliser outputs obtained with continuous adaptation are shown in Fig.7.

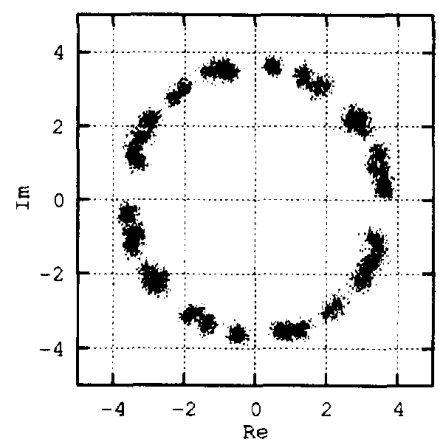

Fig.8. Outputs of CMA for 16-QAM telephone channel with non-white symbol sequence.

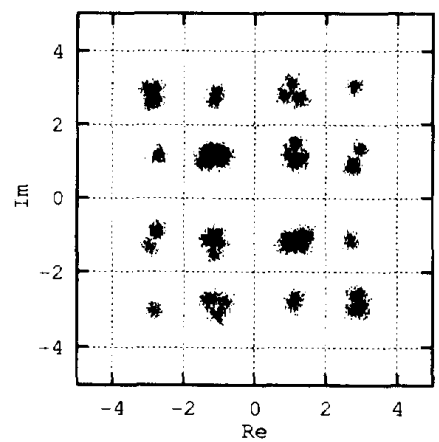

Fig.9. Outputs of two-stage blind clustering algorithm for 16-QAM telephone channel with non-white symbol sequence.

The third example investigates the performance of the two blind adaptive algorithms in the presence of non-white input $s(k)$. The telephone channel listed in Table 1 is used again and the 16-QAM symbol sequence is generated as follows. A pseudorandom binary sequence of a repetive period 256 is generated, and every four bits are converted into a 16-QAM symbol. This is designed to simulate the practical case reported in [7]. The noise power was $\sigma_{e}^{2}=-20 \mathrm{~dB}$, and the equaliser had 23 taps. For the CMA, the adaptive procedure was identical to that used in the first example. The equaliser outputs after the adaptation of 20000 samples are shown in Fig.8. where it is seen that the CMA converged to the false 
solution of the constant modulus. For the two-stage blind clustering algorithm, an adaptive gain of $\mu=0.002$ were employed for the both stages, 1500 samples were used in the stage one with a $\rho=1.6$, and 2000 samples were used in the stage two with a $\rho=0.6$. After the adaptation of 3500 samples, the equaliser outputs are plotted in Fig.9. For a sequence of the period $256 / 4=64$, it is very difficult to generate an equiprobable 16-QAM sequence. The different sizes of the clusters in Fig.9 was due to non-equiprobable 16-QAM symbol points. As expected, the distributions of the clusters were not circular. However the correct signal constellation was restored.

\section{Conclusions}

A novel multi-stage blind clustering algorithm has been presented for equalisation of high-order QAM digital communication channels. This blind adaptive algorithm adopts the criterion based on the maximum a posteriori probability density function of the equaliser output and employes a rational hierarchical decomposition of the equalisation objective. It has been shown that the multi-stage blind clustering algorithm offers significant improvement in performance over the well-known constant modulus algorithm at the cost of slightly increased computational complexity. This new blind adaptive algorithm has been shown to be very robust in the presence of non-white input symbol sequence. The current study has demonstrated that the proposed new adaptive algorithm provides a promising scheme for real-time blind equalisation of high-level signal constellations. Further investigation is warranted to establish rigorously theoretical convergence properties of the algorithm.

\section{References}

[1] Y. Sato, "A method of self-recovering equalization for multilevel amplitude-modulation systems," IEEE Trans. Communications, Vol.COM-23, pp.679-682, 1975.

[2] D. Godard, "Self-recovering equalization and carrier tracking in two-dimensional data communication systems," IEEE Trans. Communications, Vol.COM-28. pp.1867-1875, 1980.

[3] J.R. Treichler and B.G. Agee, "A new approach to multipath correction of constant modulus signals," IEEE Trans. Acoustics, Speech and Signal Processing, Vol.ASSP-31, No.2, pp.459-472, 1983.

[4] A. Benveniste and M. Goursat, "Blind Equalizers," IEEE Trans. Communications, Vol.COM-32, pp.871-883, 1984.

[5] S. Bellini and F. Rocca, "Blind deconvolution: polyspectra or Bussgang techniques?" in Digital Communications, eds., E. Biglieri and G. Prati. Amsterdam: North-Holland, 1986, pp.251-263.

[6] G. Picchi and G. Prati, "Blind equalization and carrier recovering using a "stop-and-go" decision-directed algorithm." IEEE Trans. Communications, Vol.COM-35, pp.877-887, 1987.
[7] J.R. Treichler, "Application of blind equalization techniques to voiceband and RF modems," in Preprints 4th IFAC Int. Symposium Adaptive Systems in Control and Signal Processing (France), 1992, pp.705-713.

[8] N.K. Jablon. "Joint blind equalization, carrier recovery, and timing recovery for high-order QAM signal constellations," IEEE Trans. Signal Processing, Vol.40. No.6, pp.1383-1398, 1992.

[9] G.B. Giannakis and J.M. Mendel, "Identification of nonminimum phase system using higher order statistics," IEEE Trans. Acoustics, Speech and Signal Processing, Vol.ASSP-37, pp.360-377, 1989.

[10] H.-H. Chiang and C.L. Nikias, "Adaptive deconvolution and identification of nonminimum phase FIR systems based on cumulants," IEEE Trans. Automatic Control, Vol.AC-35, pp.36-47, 1990.

[11] F.-C. Zheng, S. McLaughlin and B. Mulgrew, "Blind equalization of nonminimum phase channels: higher order cumulant based algorithm," IEEE Trans. Signal Processing, to appear, February, Vol.40, No.2, 1993.

[12] N. Seshadri. "Joint data and channel estimation using blind trellis search techniques," submitted for publication, 1991.

[13] K. Giridhar, J.J. Shynk and R.A. Iltis, "A modified Bayesian algorithm with decision feedback for blind adaptive equalization," in Preprints 4th IFAC Int. Symposium Adaptive Systems in Control and Signal Processing (France), 1992, pp.737-742.

[14] E. Zervas, J. Proakis and V. Eyuboglu, "A quantized channel approach to blind equalization," in Proc. ICC'92 (Chicago), 1992, Vol.3, pp.351.8.1-351.8.5.

[15] J. Karaoguz and S.H. Ardalan, "A soft decision-directed blind equalization algorithm applied to equalization of mobile communication channels," in Proc. ICC'92 (Chicago), 1992, Vol.3, pp.343.4.1-343.4.5.

[16] K. Abend and B.D. Fritchman, "Statistical detection for communication channels with intersymbol interference," Proc. IEEE, Vol.58, No.5, pp.779-785, 1970.

[17] S.C. Bateman and S.Y. Ameen, "Comparison of algorithms for use in adaptive adjustment of digital data receivers," IEE Proc., Pt.I, Vol.137, No.2, pp.85-96, 1990. 